\title{
The Multivariate Müntz-Szasz Problem in Weighted Banach Space on $\mathbb{R}^{n}$
}

\author{
Xiangdong Yang \\ Department of Mathematics, Kunming University of Science and Technology, Kunming, Yunnan 650093, China
}

Correspondence should be addressed to Xiangdong Yang; yangsddp@126.com

Received 19 February 2014; Accepted 1 May 2014; Published 19 May 2014

Academic Editor: Sung G. Kim

Copyright ( 2014 Xiangdong Yang. This is an open access article distributed under the Creative Commons Attribution License, which permits unrestricted use, distribution, and reproduction in any medium, provided the original work is properly cited.

The purpose of this paper is to give an extension of Müntz-Szasz theorems to multivariable weighted Banach space. Denote by $\left\{\lambda_{k}=\left(\lambda_{k}^{1}, \lambda_{k}^{2}, \ldots, \lambda_{k}^{n}\right)\right\}_{k=1}^{\infty}$ a sequence of real numbers in $\mathbb{R}_{+}^{n}$. The completeness of monomials $\left\{t^{\lambda_{k}}\right\}$ in $C_{\alpha}$ is investigated, where $C_{\alpha}$ is the weighted Banach spaces which consist of complex continuous functions $f$ defined on $\mathbb{R}^{n}$ with $f(t) \exp (-\alpha(t))$ vanishing at infinity in the uniform norm.

\section{Introduction and Notations}

The object of this paper is to obtain some completeness criteria for monomials $\left\{t^{\lambda_{k}}\right\}$, which is analogous to Müntz-Szasz theorem in one variable.

The following notations will be used. Throughout this paper, points of $\mathbb{C}^{n}$ will be denoted by $z=\left(z_{1}, \ldots, z_{n}\right)$, where $z_{k} \in \mathbb{C}$. If $z_{k}=x_{k}+i y_{k}, x=\left(x_{1}, \ldots, x_{n}\right), y=\left(y_{1}, \ldots, y_{n}\right)$, then we write $z=x+i y$. The vectors $x=\mathfrak{R} z$ and $y=\mathfrak{\Im} z$ are the real and imaginary parts of $z$, respectively, and $\mathbb{R}^{n}$ will be thought of as the set of all $z \in \mathbb{C}^{n}$ with $\Im z=0$, furthermore; $\mathbb{R}_{+}^{n}=\left\{x=\left(x_{1}, x_{2}, \ldots, x_{n}\right) \mid x_{j}>0\right.$ for all $\left.1 \leq j \leq n\right\}$, and $\mathbb{C}_{+}^{n}=\left\{z=\left(z_{1}, z_{2}, \ldots, z_{n}\right) \mid \mathfrak{R} z_{j}>0\right.$ for all $\left.1 \leq j \leq n\right\}$. The set of nonnegative integers will be denoted by $\mathbb{Z}_{+}$. The notations

$$
\begin{gathered}
|z|=\left(\left|z_{1}\right|^{2}+\cdots+\left|z_{n}\right|^{2}\right)^{1 / 2} \\
|\Re z|=\left(\left|x_{1}\right|^{2}+\cdots+\left|x_{n}\right|^{2}\right)^{1 / 2} \\
|\Im z|=\left(\left|y_{1}\right|^{2}+\cdots+\left|y_{n}\right|^{2}\right)^{1 / 2} \\
z^{\beta}=z_{1}^{\beta_{1}} \cdots z_{n}^{\beta_{n}} \\
\langle z, t\rangle=z_{1} t_{1}+\cdots+z_{n} t_{n}
\end{gathered}
$$

will be used for any multi-index $\beta$ and any $t \in \mathbb{R}^{n}$. The unit ball of $\mathbb{C}^{n}$ will be denoted by $\mathbb{B}_{n}=\left\{z \in \mathbb{C}^{n}:|z|<1\right\}$.

By a complete system of elements $\left\{h_{k}\right\}$ of a Banach space $B$, we mean $\overline{\operatorname{Span}}\left\{h_{k}\right\}=B$; that is, the completeness is equivalent to the possibility of an arbitrary good approximation of any element of the space by linear combination of elements of this system.

The famous Müntz-Szasz theorem asserts that given a sequence of real numbers $0<\lambda_{1}<\lambda_{2}<\cdots$ the functions $\left\{1, t^{\lambda_{1}}, t^{\lambda_{2}}, \cdots\right\}$ are complete $C[0,1]$ if and only if

$$
\sum_{k=1}^{\infty} \frac{1}{\lambda_{k}}=\infty
$$

This classical result inspired an intensive research of related questions. Via duality, making use of suitable analytic varieties in the polydisk, in [1], for $1 \leq p_{1}<p_{2}<\infty$ and $n \geq 2$, it is shown that there exists a sequence of monomials $\left\{t^{\lambda_{k}}\right\}$ with $\lambda_{k}^{j} \sim k$ for each $j=1,2, \ldots, n$ whose linear span is dense in $L^{p_{1}}\left(I^{n}\right)$ but not in $L^{p_{2}}\left(I^{n}\right)$, where $I^{n}$ is the Cartesian product of $n$ copies of the closed unit interval $[0,1]$. The MüntzSzasz theorem is extended to multivariables and more general results are obtained by replacing $t^{\lambda_{k}}$ by $\psi(t)^{\lambda_{k}}$ for some function $\psi(t)$ in [2]. For $\Omega \subset \mathbb{R}^{n}$, the so-called Müntz set relative to $\Omega$ is defined in [3], which enables one to construct "optimally sparse" lattice points sets for which density holds.

It is a natural goal to consider whether it could give completeness conditions analogous to Müntz-Szasz theorem in the weighted higher-dimensional Banach space on $\mathbb{R}^{n}$ case. The paper is concerned with this problem. 
Let $\alpha(t)$ be a nonnegative continuous function defined on $\mathbb{R}^{n}$, henceforth, called a weight, satisfying

$$
\lim _{|t| \rightarrow \infty} \frac{\alpha(t)}{\log |t|}=\infty
$$

Given a weight $\alpha(t)$, the weighted Banach space $C_{\alpha}$ consists of complex continuous functions $f$ defined on $\mathbb{R}^{n}$ with $f(t) \exp (-\alpha(t))$ vanishing at infinity, normed by

$$
\|f\|_{\alpha}=\sup \left\{|f(t) \exp (-\alpha(t))|: t \in \mathbb{R}^{n}\right\}
$$

Our space $C_{\alpha}$ is rooted from $[4-9,12,13]$, in which the exponential polynomial approximation problem is investigated.

Motivated by the Bernstein problem and the Müntz theorem in [10], combining Malliavin's uniqueness theorem in [11], in his paper [12], Guantie Deng obtained a necessary and sufficient condition for the functions $\left\{1, t^{\lambda_{1}}, t^{\lambda_{2}}, \ldots\right\}$ to be dense in $C_{\alpha}$. The result which initiated the investigation of Müntz problem on weighted Banach space consists of complex functions continuous on the real axis and is described below.

Theorem 1. Suppose $\alpha(t)$ is an even function satisfying (3) and $\alpha\left(e^{t}\right)$ is a convex function on $\mathbb{R}$. Let $\Lambda=\left\{\lambda_{k}: k=1,2, \ldots\right\}$ be a sequence of strictly increasing positive integers and let

$$
\Lambda(r)=2 \sum_{\lambda_{k} \leq r} \frac{1}{\lambda_{k}}, \quad \text { if } r \geq \lambda_{1} ; \lambda(r)=0 \text {, otherwise, }
$$

$k(r)=\Lambda(r)-\log ^{+} r, \log ^{+} r=\max \{\log r, 0\}, \widetilde{k}(r)=\inf \left\{k\left(r^{\prime}\right):\right.$ $\left.r^{\prime} \geq r\right\}$. If

$$
\int_{0}^{+\infty} \frac{\alpha(\exp \{\tilde{k}(t)-a\})}{1+t^{2}} d t=\infty
$$

for each $a \in \mathbb{R}$, then $\operatorname{Span}\left\{1, t^{\lambda_{1}}, t^{\lambda_{2}}, \ldots\right\}$ is dense in $C_{\alpha}$.

Conversely, if the sequence $\Lambda$ contains all of the odd integers, then, for $\operatorname{Span}\left\{1, t^{\lambda_{1}}, t^{\lambda_{2}}, \ldots\right\}$ to be dense in $C_{\alpha}$, it is necessary that (6) holds for each $a \in \mathbb{R}$.

Deng's result was generalized to the case where the weighted Banach space consists of complex functions continuous on infinitely many disjoint closed intervals in [7]. The result is described as follows.

Let $E$ be a union of infinitely many disjoint closed intervals:

$$
\begin{gathered}
E=\bigcup_{k=1}^{\infty} I_{k}, \\
I_{k}=\left[a_{k}, b_{k}\right], \quad 0<a_{1}<b_{1}<a_{2}<b_{2}<\cdots<b_{k},
\end{gathered}
$$

where $I_{k}$ satisfies $\operatorname{dist}\left(0, I_{k}\right) \rightarrow \infty$.
Theorem 2. Suppose $\alpha(t)$ is defined by (3) and $\Lambda=\left\{\lambda_{k}: k=\right.$ $1,2, \ldots\}$ is a sequence of complex numbers satisfying the following conditions:

$$
\begin{gathered}
\text { the } \lambda_{k} \text { are all distinct and } \lim _{k \rightarrow \infty}\left|\lambda_{k}\right|=\infty, \\
\lim _{k \rightarrow \infty} \frac{k}{\left|\lambda_{k}\right|}=D, \quad(0<D<\infty), \\
\left|\arg \left(\lambda_{k}\right)\right|<\beta<\frac{\pi}{2} .
\end{gathered}
$$

Let

$$
h=\frac{1}{\eta}+\varepsilon_{0},
$$

where $\varepsilon_{0}$ is some positive number and

$$
\eta=\max _{0<\delta<D \cos \beta} \frac{2 \delta}{\sqrt{D^{2} \sin ^{2} \beta+\delta^{2}}}(D \cos \beta-\delta) .
$$

If

$$
\int_{E} \alpha(t) \omega\left(i, d t, \frac{\mathbb{C}}{E}\right)=+\infty
$$

where $\omega(i, d t, \mathbb{C} \backslash E)$ is the harmonic measure for the domain $\mathbb{C} \backslash E$ as seen from $i$ and if

$$
\int^{\infty} \frac{\alpha(t)}{t^{1+h}} d t=+\infty
$$

then the system $\left\{t^{\lambda_{k}}\right\}(k=1,2, \ldots)$ is complete in $C_{0}(E)$.

Motivated by $[4-9,12,13]$, in this paper, we will investigate the completeness of monomials $\left\{t^{\lambda_{k}}\right\}$ in $C_{\alpha}$, where $\left\{\lambda_{k}=\right.$ $\left.\left(\lambda_{k}^{1}, \lambda_{k}^{2}, \ldots, \lambda_{k}^{n}\right)\right\}_{k=1}^{\infty}$ is a sequence of real numbers in $\mathbb{R}_{+}^{n}$ and $\alpha(t)$ is a nonnegative continuous function defined in $\mathbb{R}^{n}$ for $t \in \mathbb{R}^{n}$. Our result can be thought of as a generalization of the results in $[7,8,13]$ to multivariable case. It also can be regarded as a generalization of the results in [1-3]. Our main result depends upon the uniqueness theory of analytic functions on the unit ball $\mathbb{B}_{n}$. As is well known the zeros of analytic functions in $\mathbb{C}^{n}(n \geq 2)$ are never discrete. The multivariable case may be different from a single variable case. That is why it needs to be treated separately (see [9]).

In the sequel, we will use $A$ to denote positive constants that may vary in value from one occurrence to the next. The main results of this paper are as follows.

Theorem 3. Let $\beta(s)$ be a nonnegative and nondecreasing function with continuous derivative defined on $(A,+\infty)$ for some positive constant $A$, satisfying

$$
\int_{A}^{+\infty} \frac{\beta(s)}{s^{2}} d s=+\infty,
$$

and let $\alpha(t)$ be a nonnegative continuous function defined on $\mathbb{R}^{n}$ satisfying

$$
A_{1}\left(\sum_{j=1}^{n}\left(\log \left|t_{j}\right|\right)^{2}\right) \leq \int_{A_{2}}^{|t|} \frac{\beta(s)}{s} d s \leq \alpha(t),
$$


where $A_{1}$ and $A_{2}$ are fixed positive constants. Let $\left\{\lambda_{k}=\left(\lambda_{k}^{1}\right.\right.$, $\left.\left.\lambda_{k}^{2}, \ldots, \lambda_{k}^{n}\right)\right\}_{k=1}^{\infty}$ be a sequence of real numbers in $\mathbb{R}_{+}^{n}$. If

$$
\sum_{k=1}^{\infty}\left|\frac{2 \lambda_{k}^{j}-\lambda_{k}^{1}-1}{2 \lambda_{k}^{j}}\right|^{2+\varepsilon_{0}}=+\infty
$$

for some $j \in\{2, \ldots, n\}$, where $\varepsilon_{0}$ is some fixed positive constant, then $\left\{t^{\lambda_{k}}\right\}$ is complete in $C_{\alpha}$.

Theorem 4. Let $\alpha(t)$ be a nonnegative continuous function defined on $\mathbb{R}^{n}$ satisfying

$$
\alpha(t) \leq A(\log |t|)^{2},
$$

where $A$ is some fixed positive constant. Suppose that $\left\{\lambda_{k}=\right.$ $\left.\left(\lambda_{k}^{1}, \lambda_{k}^{2}, \ldots, \lambda_{k}^{n}\right)\right\}_{k=1}^{\infty}$ is a sequence of real numbers in $\mathbb{R}_{+}^{n}$. If

$$
\sum_{k=1}^{\infty} \frac{1}{\left(\lambda_{k}^{j}\right)^{2+\varepsilon}}<+\infty
$$

is satisfied for every $j \in\{1,2, \ldots, n\}$ and arbitrary positive constant $\varepsilon$, then $\left\{t^{\lambda_{k}}\right\}$ is incomplete in $C_{\alpha}$.

There are obvious ways in which our main result can be generalized: the example of Theorem $1 \mathrm{can}$ be extended to much more general sets by using Lemma 5 in Section 2 . We decided not to pursue elaborations; our aim is to present the essence of an interesting qualitative phenomenon, avoiding as far as possible obscuring technicalities.

The remaining part of this paper is organized as follows. In Section 2 we give some notation and we introduce several results used later. In Section 3 we prove our main results.

\section{Preliminaries}

In this section, we will establish a uniqueness result for functions holomorphic in $\mathbb{B}^{n}$. The proof of such a result depends on several lemmas.

Following [14], we denote the angles $(\alpha \leq \arg \leq \beta)$ and $(\alpha<\arg <\beta)$ by $[\alpha, \beta]$ and $(\alpha, \beta)$, respectively. Let a function $f(z)$ be analytic in $(\alpha, \beta)$ and continuous in $[\alpha, \beta]$, and let the relations

$$
\max _{\alpha \leq \theta \leq \beta}\left|f\left(r e^{i \theta}\right)\right|<\exp \left(r^{v}\right), \quad r>r_{v}
$$

hold for some $v>0$. Denote by $\rho$ the most lower bound of all $v_{*}>0$ such that

$$
\limsup _{r \rightarrow \infty} r^{-v_{*}} \log \left|f\left(r e^{i \theta}\right)\right| \equiv 0, \quad \alpha \leq \theta \leq \beta .
$$

Then, the number $\rho$ is called the order of the function $f(z)$. Recall that the canonical Weierstrass factor is defined by

$$
\begin{gathered}
E(z, q)=1-z, \quad q=0, \\
E(z, q)=(1-z) \exp \left(z+\frac{z^{2}}{2}+\cdots+\frac{z^{q}}{q}\right), \quad q=1,2 \ldots
\end{gathered}
$$

The canonical Nevanlinna factor is defined by

$$
D_{q}\left(z, a_{k}\right)=\frac{E\left(z / a_{k}, q\right)}{E\left(z / \overline{a_{k}}, q\right)} \text {. }
$$

We define the following modified canonical factor by

$$
\begin{gathered}
E^{-}\left(\frac{z}{a_{k}}, q\right)=1-\frac{z}{a_{k}}, \quad q=0, \\
E^{-}\left(\frac{z}{a_{k}}, q\right)=\left(1-\frac{z}{a_{k}}\right) \exp \left(\frac{(-1) z}{a_{k}}+(-1)^{2} \frac{\left(z / a_{k}\right)^{2}}{2}\right. \\
\left.+\cdots+(-1)^{q} \frac{\left(z / a_{k}\right)^{q}}{q}\right)
\end{gathered}
$$

for $q=1,2 \ldots$,

$$
\begin{aligned}
E^{+} & \left(\frac{z}{a_{k}}, q\right)=1+\frac{z}{a_{k}}, \quad q=0, \\
E^{+}\left(\frac{z}{a_{k}}, q\right)= & \left(1+\frac{z}{a_{k}}\right) \\
& \times \exp \left(\frac{z}{a_{k}}+\frac{\left(z / a_{k}\right)^{2}}{2}+\cdots+\frac{\left(z / a_{k}\right)^{q}}{q}\right)
\end{aligned}
$$

for $q=1, \ldots$, and

$$
D_{q}^{ \pm}\left(z, a_{k}\right)=\frac{E^{-}\left(z / a_{k}, q\right)}{E^{+}\left(z / \overline{a_{k}}, q\right)}
$$

From page 25 of [14], we know that an analytic function of arbitrary finite order admits canonical representation as follows.

Lemma 5. Every function $f(z)$ analytic and of a finite order $\rho$ in the right half plane $\Re z>0$ admits the representations

$$
\begin{aligned}
f(z)= & e^{\left(b_{0}+b_{1} z+\cdots+b_{q} z^{q}\right)} \prod_{\left|a_{k}\right| \leq 1} \frac{z-a_{k}}{z+\overline{a_{k}}} \prod_{\left|a_{k}\right|>1} D_{q}^{ \pm}\left(z, a_{k}\right) \\
& \times \exp \left\{\frac{1}{\pi} \int_{-\infty}^{+\infty} \frac{(i t z+1)^{q+1} \log |f(i t)|}{\left(t^{2}+1\right)^{q+1}(t-i z)} d t\right. \\
& \left.+\frac{1}{\pi} \int_{-\infty}^{+\infty} \frac{(i t z+1)^{q+1} d \varphi(t)}{\left(t^{2}+1\right)^{q+1}(t-i z)}\right\},
\end{aligned}
$$

where $q=[\rho], b_{0}, \ldots, b_{q}$ are complex numbers, $a_{k}=r_{k} e^{i \theta_{k}}$, $-\pi / 2<\theta_{k}<\pi / 2$ are zeros of $f(z)$, and $\varphi(t)$ is a singular 
boundary function. All integrals and infinite products are absolutely convergent. The following relations hold:

$$
\begin{gathered}
\sum_{r_{k} \leq 1} r_{k} \cos \theta_{k}<\infty, \quad \sum_{r_{k}>1} r_{k}^{-v-\varepsilon} \cos \theta_{k}<\infty, \\
\int_{-\infty}^{+\infty} \frac{\log |f(i t)|}{1+|t|^{1+v+\varepsilon}} d t<\infty, \\
\int_{-\infty}^{+\infty} \frac{|d \varphi(t)|}{1+|t|^{1+v+\varepsilon}}<\infty,
\end{gathered}
$$

where $v=\max (\rho, 1)$ and $\varepsilon$ is an arbitrary positive number.

We can deduce the following lemma by conformal maps.

Lemma 6. Suppose that $f(z)$ is analytic in the unit disk $\mathbb{D}=$ $\{|z|<1\}$, satisfying

$$
|f(z)| \leq \exp \left\{A(1-|z|)^{-\rho}\right\},
$$

for some $\infty>\rho>1$. If, for some fixed $\varepsilon_{0}>0$,

$$
\sum_{k=1}^{+\infty}\left(\frac{1-a_{k}}{1+a_{k}}\right)^{\rho+\varepsilon_{0}}=+\infty
$$

and $f\left(a_{k}\right)=0$, where $\left\{a_{k}\right\}_{k=1}^{\infty}$ is a sequence of real numbers in $\mathbb{D}=\{|z|<1\}$, then, $f(z) \equiv 0$.

Proof. We will show that the existence of some $f(z)$ satisfying (28) and $f\left(a_{k}\right)=0$ contradicts Lemma 5.

Suppose that $f(z)$ is a nontrivial function analytic in the unit disk, satisfying (28) and $f\left(a_{k}\right)=0$. Taking the conformal transformation $z=(w-1) /(w+1)$, it is well known that such transformation maps the unit disk onto the right half plane. Thus,

$$
|f(z)|=\left|f\left(\frac{w-1}{w+1}\right)\right| \leq A \exp \left\{A\left(1-\left|\frac{w-1}{w+1}\right|\right)\right\}^{-\rho} .
$$

Define

$$
g(w)=f\left(\frac{w-1}{w+1}\right) ;
$$

then, we get a function $g(w)$ which is analytic in the right half plane $\mathbb{C}_{+}=\{\Re w>0\}$ and satisfies $g\left(\lambda_{k}\right)=0$ for $\lambda_{k}=$ $\left(1+a_{k}\right) /\left(1-a_{k}\right)>0$; furthermore, we have

$$
|g(w)| \leq A e^{A|w|^{p}}
$$

for sufficient large $|w|$. Applying Lemma 5, we have

$$
\sum_{k=1}^{+\infty} \frac{1}{\lambda_{k}^{\rho+\varepsilon}}<+\infty
$$

for arbitrary $\varepsilon>0$; thus,

$$
\sum_{k=1}^{+\infty}\left(\frac{1-a_{k}}{1+a_{k}}\right)^{\rho+\varepsilon}<+\infty
$$

which is a contradiction to (29).
The following uniqueness lemma is crucial in the establishment of the main result of this paper. It is closely related to results of [15].

Lemma 7. Let $\left\{a_{k}=\left(a_{k}^{1}, a_{k}^{2}, \ldots, a_{k}^{n}\right)\right\}_{k=1}^{\infty}$ be a sequence in $\mathbb{R}^{n}$, satisfying $0<\left|a_{k}^{j}\right|<1$ for all $k$ and $j=1,2, \ldots, n$. Suppose that

$$
\sum_{k=1}^{+\infty}\left|\frac{a_{k}^{j}-1}{a_{k}^{j}}\right|^{\rho+\varepsilon_{0}}=+\infty
$$

for some $j \in\{1,2, \ldots, n\}$, where $\varepsilon_{0}$ is some fixed positive constant. Let $E$ be the set of all $z \in \mathbb{B}_{n}$ that have $z_{j} \in\left\{a_{k}^{j}\right\}$. Let $f(z)$ be an analytic function on $\mathbb{B}_{n}$ which satisfies the growth condition

$$
|f(z)| \leq \exp \left\{A(1-|z|)^{-\rho}\right\}
$$

for some $0<A<\infty$ and $\infty>\rho>1$. Denote by $Z(f)$ the zero set of $f(z)$. If $Z(f) \supset E$, then $f \equiv 0$.

Proof. We will follow the proof of Theorem 7.3.4 on pages 135-136 of [15]. Without loss of generality, it is enough to investigate the case where $j=1$.

Denote, by $w=\left(w_{1}, w^{\prime}\right), w^{\prime}=\left(w_{2}, \ldots, w_{n}\right)$. Let $\Omega$ be the set of all $w \in \mathbb{B}_{n}$ satisfying

$$
\left|2 w_{1}-1\right|<1, \quad\left|w^{\prime}\right|<\left|1-w_{1}\right| .
$$

It is apparent that $\Omega$ is a nonempty set. Thus, it is enough to prove that $f \equiv 0$ for fixed $w \in \Omega$.

Define

$$
h(z)=\left(\frac{1+z}{2}, \frac{1-z}{2\left(1-w_{1}\right)} w^{\prime}\right),
$$

where $z=r e^{i \theta} \in \mathbb{D}$. Let $\delta=1-\left|w^{\prime}\right|^{2}\left|1-w_{1}\right|^{2}$. Then, $0<\delta<1$ and

$$
\begin{aligned}
4\left(1-\left|h\left(r e^{i \theta}\right)\right|^{2}\right) & =2\left(1-r^{2}\right)+\delta\left(1-2 r \cos \theta+r^{2}\right) \\
& \geq 2\left(1-r^{2}\right),
\end{aligned}
$$

for $0 \leq r<1$ and $|\theta| \leq \pi$. It follows that $h$ maps $\mathbb{D}$ into $\mathbb{B}_{n}$. Define

$$
g(z)=f(h(z)), \quad z \in \mathbb{D} .
$$

By (36) and (39), we have

$$
\begin{aligned}
\log \left|g\left(r e^{i \theta}\right)\right| & \leq \frac{A}{\left(1-\left|h\left(r e^{i \theta}\right)\right|^{2}\right)^{\rho}} \\
& \leq \frac{A}{(1-|z|)^{\rho}} .
\end{aligned}
$$

Note that

$$
g\left(2 a_{k}^{1}-1\right)=f\left(a_{k}^{1}, \frac{1-a_{k}^{1}}{1-w_{1}} w^{\prime}\right) .
$$


Since $Z(f) \supset E$, the zero sequence $\left\{2 a_{k}^{1}-1\right\}_{k=1}^{\infty}$ satisfies (35) which is (29) in Lemma 6. It follows that $g(z)=0$ for all $z \in$ $\mathbb{D}$. In particular, $2 w_{1}-1 \in \mathbb{D}$, so that $f(w)=f\left(w_{1}, w^{\prime}\right)=$ $g\left(2 w_{1}-1\right) \equiv 0$.

We will be concerned with density of polynomials in $C_{\alpha}$ which is essential in the proof of Theorem 3. We need the following result from [16] (see also similar result in [17]).

Lemma 8. Let $\beta(s)$ be a nonnegative and nondecreasing function with continuous derivative defined on $(A,+\infty)$ for some positive constant A, satisfying

$$
\int_{A}^{\infty} \frac{\beta(s)}{s^{2}} d s=\infty
$$

If $\mu$ is a complex measure on $\mathbb{R}^{n}$ such that

$$
\int_{|t|>A} \exp \left(\int_{A}^{|t|} \frac{\beta(s)}{s} d s\right) d \mu(t)<\infty,
$$

then the polynomials are dense in $L_{1}\left(\mathbb{R}^{n}, \mu\right)$.

Proof. Since a real measure has the Jordan decomposition as the difference of positive and negative variation (see page 119 of [18], e.g.), for any complex measure $\mu$ on a $\sigma$-algebra in $X$, there is a measurable function $h$ such that $|h(x)|=1$ for all $x \in X$ and such that $d \mu=h d|\mu|$ (see page 124 of [18], e.g.), replacing the positive measure in the proof of Theorems 2.1 and 2.3 in [16]; repeating the proof there word by word, we can see that the same conclusion still holds for the case of complex measures.

We will use the following elementary results on inequality in [19].

Lemma 9. Let $a_{j} \geq 0$ for $j=1, \ldots, n$. Then,

$$
\left(\sum_{j=1}^{n} a_{j}\right)^{p} \leq n^{p-1}\left(\sum_{k=1}^{n} a_{j}^{p}\right)
$$

for $1 \leq p<\infty$

Lemma 10. Let

$$
\log ^{+} a= \begin{cases}\log a, & a \geq 1 \\ 0, & 0 \leq a<1 .\end{cases}
$$

If $a_{j} \geq 0$ for $j=1, \ldots, n$, then,

$$
\log ^{+}\left(\sum_{j=1}^{n} a_{j}\right) \leq \sum_{j=1}^{n} \log ^{+} a_{j}+A
$$

where $A$ is some positive constant depending on $n$.

\section{Proof of Main Results}

In this section, we prove the main results of this paper.

Proof of Theorem 3. If $\left\{t^{\lambda_{k}}\right\}$ is incomplete in $C_{\alpha}$, by the HahnBanach theorem there exists a nontrivial bounded linear functional $T$ such that $\|T\|=1$ and $T\left(t^{\lambda_{k}}\right)=0$. So by the Riesz representation theorem, there exists a complex measure $\mu$ on $\mathbb{R}^{n}$ satisfying

$$
\begin{gathered}
\|\mu\|=\int_{\mathbb{R}^{n}} e^{\alpha(t)}|d \mu(t)|=\|T\|, \\
T(h)=\int_{\mathbb{R}^{n}} h(t) d \mu(t), \quad h \in C_{\alpha} .
\end{gathered}
$$

Define

$$
\begin{aligned}
f(z)= & \int_{\mathbb{R}^{n}} t^{z} d \mu(t) \\
= & \int_{-\infty}^{0} \cdots \int_{-\infty}^{0}\left|t_{1}\right|^{z_{1}} e^{-i(\pi / 2) z_{1}} \cdots\left|t_{n}\right|^{z_{n}} e^{-i(\pi / 2) z_{n}} d \mu(t) \\
& +\cdots+\int_{0}^{+\infty} \cdots \int_{0}^{+\infty} t_{1}^{z_{1}} e^{i(\pi / 2) z_{1}} \cdots t_{n}^{z_{n}} \\
& \times e^{-i(\pi / 2) z_{n}} d \mu(t) ;
\end{aligned}
$$

then, $f(z)$ is holomorphic in $\mathbb{H}=\left\{z: \mathfrak{R} z_{1}>\left|z^{\prime}\right|^{2}\right\} \bigcap \mathbb{C}_{+}^{n}$ and satisfies

$$
\begin{aligned}
|f(z)| \leq 2^{n} \int_{\mathbb{R}^{n}} \exp \left(\left(\sum_{j=1}^{n} x_{j} \log \left|t_{j}\right|\right)-\alpha(t)\right) \\
\quad \times \exp \left(\sum_{j=1}^{n} \frac{\pi}{2}\left|y_{j}\right|\right) \exp (\alpha(t)) d \mu(t) ;
\end{aligned}
$$

thus, by (14), we have

$$
\begin{aligned}
& \sup _{t \in \mathbb{R}^{n}}\left\{\left(\sum_{j=1}^{n} x_{j} \log \left|t_{j}\right|\right)-\alpha(t)\right\} \\
& \leq \sum_{j=1}^{n} \sup _{t_{j} \in \mathbb{R}}\left\{x_{j} \log \left|t_{j}\right|-A_{1}\left(\log \left|t_{j}\right|\right)^{2}\right\}
\end{aligned}
$$

which yields

$$
|f(z)| \leq 2^{n}\|T\| \exp \left\{A|\Re z|^{2}+\sum_{j=1}^{n} \frac{\pi}{2}\left|y_{j}\right|\right\},
$$

for all $z \in \mathbb{M}$. Denote by $e^{j}=\underbrace{(0, \ldots, 1, \ldots, 0)}_{j}$ the Cayley transform from $\mathbb{U}$ to $\mathbb{B}_{n}$; consider

$$
w=\varphi(z)=\frac{2 z}{1+z_{1}}-e^{1} .
$$

Define the function

$$
g(w)=f\left(\varphi^{-1}(w)\right)
$$


where $f$ is the function defined in (52) and $\varphi^{-1}$ is the inverse of the Cayley transform defined in (53). Thus, we have

$$
|g(w)|=\left|f\left(\varphi^{-1}(w)\right)\right| \leq A \exp \left\{\frac{A}{(1-|w|)^{2}}\right\} .
$$

Denote, by

$$
\begin{gathered}
a_{k}^{1}=\frac{2 \lambda_{k}^{1}}{1+\lambda_{k}^{1}}-1, \\
a_{k}^{j}=\frac{2 \lambda_{k}^{j}}{1+\lambda_{k}^{j}},
\end{gathered}
$$

for $j=2, \ldots, n$. It is obvious that (15) relates to (35) in Lemma 7. Thus, we have $f(z) \equiv 0$; it follows that $T\left(t^{j}\right)=0$ for all nonnegative integers $j=\left(j_{1}, \ldots, j_{n}\right)$. From Lemma 8 , it is obvious that $T \equiv 0$, from which the conclusion of Theorem 3 follows.

Proof of Theorem 4. If there exist a real constant $A$ and some positive constant $\varepsilon_{0}$ such that both (16) and (17) are satisfied, we will show the existence of a nontrivial bounded functional which annihilates $\left\{t^{\lambda_{k}}\right\}$.

If (17) holds, we know that there exists analytic functions $g_{j}\left(z_{j}\right)(j=1,2, \ldots, n)$ which can be represented in the forms in Lemma 5. The function $g_{j}\left(z_{j}\right)(j=1,2, \ldots, n)$ verifies $g_{j}\left(\lambda_{k}^{j}\right)=0$ for all $\lambda_{k}^{j}$ satisfying (17); furthermore, the following estimate

$$
\left|g_{j}\left(z_{j}\right)\right| \leq e^{A_{1}\left(\left|x_{j}\right|^{2}+\left|y_{j}\right|^{2}\right)}
$$

holds for all $z_{j} \in \mathbb{C}_{+}$. It is obvious that

$$
\exp \left(\left\langle z, e^{j}\right\rangle^{2}\right)=\exp \left(z_{j}^{2}\right) .
$$

For $z \in \mathbb{C}_{+}^{n}$, define

$$
\begin{gathered}
G_{0}(z)=\prod_{j=1}^{n} g_{j}\left(z_{j}\right)=\prod_{j=1}^{n} g_{j}\left(\left\langle z, e^{j}\right\rangle\right) \\
G_{1}(z)=G_{0}(z) \exp \left(A_{2} \sum_{j=1}^{n}\left(\left\langle z, e^{j}\right\rangle\right)^{2}\right),
\end{gathered}
$$

where $A_{2}>A_{1}$ is some positive constant satisfying

$$
A-\frac{1}{4\left(A_{1}+A_{2}\right)}<0
$$

for the constant $A$ defined in (16). Then, we have the following estimate:

$$
\begin{array}{r}
\left|G_{1}(z)\right| \leq \exp \left\{\left(A_{1}+A_{2}\right)\left(\sum_{j=1}^{n} x_{j}^{2}\right)-\left(A_{1}-A_{2}\right)\left(\sum_{j=1}^{n} y_{j}^{2}\right)\right\}, \\
z \in \mathbb{C}_{+}^{n} .
\end{array}
$$

Suppose that $G_{1}(z)$ is the analytic function defined in (59); define

$$
h_{0}(t)=\int_{\mathbb{R}^{n}} G_{1}\left(\frac{\mathbf{1}}{\mathbf{2}}+i y\right) t^{-(\mathbf{1} / \mathbf{2}+i y)} d m_{n}(y), \quad t \in \mathbb{R}_{+}^{n},
$$

where $\mathbf{1} / \mathbf{2}=(1 / 2,1 / 2, \ldots, 1 / 2)$ and $m_{n}$ denotes the Lebesgue of $\mathbb{R}^{n}$. Note first that $G_{1}(\mathbf{1} / \mathbf{2}+i y)$ is in $L^{1}\left(\mathbb{R}^{n}\right)$ by $A_{1}<A_{2}$ and (61); furthermore, $h_{0}(t)$ is continuous on $\mathbb{R}_{+}^{n}$.

Next, we claim that the integral

$$
\int_{-\infty}^{+\infty} G_{1}\left(\zeta+i \eta, z_{2}, \ldots, z_{n}\right) t^{-(\zeta+i y)} d \eta
$$

is independent of $\zeta$ for arbitrary $t=\left(t_{1}, \ldots, t_{n}\right) \in \mathbb{R}_{+}^{n}$ and complex $z=\left(z_{1}, \ldots, z_{n}\right)$. Without loss of generality, we prove it for the first coordinate. To see this, let $\Gamma$ be a rectangular path in the $\zeta+i \eta$-plane, with one edge on the image axis and one on the line $\zeta=\zeta_{1}$, whose horizontal edges move off to infinity. By Cauchy's theorem, the integral of the integrand (63) over $\Gamma$ is 0 . From (61) we know that the contribution of the horizontal edges to this integral is also 0 . Thus, it follows that (63) is the same for $\zeta=\zeta_{1}$ as for $\zeta=1 / 2$, which establishes our claim.

The same can be done for the other coordinates. Hence, we conclude from (62) that

$$
h_{0}(t)=\int_{\mathbb{R}^{n}} G_{1}(x+i y) t^{-(x+i y)} d m_{n}(y)
$$

for every $y \in \mathbb{R}^{n}$ and $x=\left(x_{1}, \ldots, x_{n}\right) \in \mathbb{R}_{+}^{n}$. From (61) and (64), we have

$$
\left|h_{0}(t)\right| \leq A_{4} e^{\left(A_{1}+A_{2}\right)\left(\sum_{j=1}^{n} x_{j}^{2}-x_{j} \log \left|t_{j}\right|\right)},
$$

where $A_{4}=\int_{\mathbb{R}^{n}} e^{-\left(A_{2}-A_{1}\right)|y|^{2}} d m_{n}(y)$. Thus,

$$
\left|h_{0}(t)\right| \leq A_{4} \prod_{j=1}^{n} e^{\inf \left\{\left(A_{1}+A_{2}\right) x_{j}^{2}-x_{j} \log \left|t_{j}\right|: x_{j} \in \mathbb{R}_{+}\right\}} ;
$$

direct calculation yields

$$
\left|h_{0}(t)\right| \leq A_{4} e^{-\left(\sum_{j=1}^{n}\left(\log \left|t_{j}\right|\right)^{2} / 4\left(A_{1}+A_{2}\right)\right)} .
$$

From (67) we know that $h_{0}(t)$ is in $L^{1}\left(\mathbb{R}^{n}\right)$. Taking the inverse Fourier transform in (64), we obtain

$$
G_{1}(z)=\int_{\mathbb{R}_{+}^{n}} h_{0}(t) t^{z} d m_{n}(t),
$$

for $x \in \mathbb{R}_{+}^{n}$. Extend $h_{0}(t)$ to an even function by defining $h_{0}(t)=h_{0}(-t)$ whenever $t_{j}<0$.

It is apparent that, for $\left|t_{j}\right| \geq 1$, we have $\log ^{+}\left|t_{j}\right|=\log \left|t_{j}\right|$. Thus, combining Lemmas 9 and 10 with (16), we conclude that there exists some positive constant $A$ such that

$$
\alpha(t) \leq A \sum_{j=1}^{n}\left(\log \left|t_{j}\right|\right)^{2} .
$$


Therefore, from (69) and (67), if (60) holds, choosing $A_{2}$ satisfying

$$
A-\frac{1}{4\left(A_{1}+A_{2}\right)}<0,
$$

we obtain the bounded linear functional

$$
T(h)=\int_{\mathbb{R}^{n}} h_{0}(t) h(t) d m_{n}(t), \quad h \in C_{\alpha}
$$

satisfying $T\left(t^{\lambda_{k}}\right)=0$ for $\left\{\lambda_{k}=\left(\lambda_{k}^{1}, \lambda_{k}^{2}, \ldots, \lambda_{k}^{n}\right)\right\}_{k=1}^{\infty}$, satisfying (17) and

$$
\|T\|=\int_{\mathbb{R}^{n}} e^{\alpha(t)}|d \mu(t)|>0 .
$$

\section{Conflict of Interests}

The author declares that there is no conflict of interests regarding the publication of this paper.

\section{Acknowledgments}

This work was done when Xiangdong Yang visited the IMA (Institute of Mathematics and its Applications) in Minnesota, USA. The author gratefully acknowledges the support from the CSC (Grant no. [2010] 5013). The author is also grateful to the IMA for partial financial support during his stay. This work is supported by the National Natural Science Foundation of China (no. 11261024).

\section{References}

[1] S. Hellerstein, "Some analytic varieties in the polydisc and the Müntz-Szasz problem in several variables," Transactions of the American Mathematical Society, vol. 158, pp. 285-292, 1971.

[2] S. Ogawa and K. Kitahara, "An extension of Müntz's theorems in multivariables," Bulletin of the Australian Mathematical Society, vol. 36, pp. 375-387, 1987.

[3] A. Kroó, "A geometric approach to the multivariate Müntz problem," Proceedings of the American Mathematical Society, vol. 121, pp. 199-208, 1994.

[4] G. T. Deng, "Incompleteness and closure of a linear span of exponential system in a weighted Banach space," Journal of Approximation Theory, vol. 125, no. 1, pp. 1-9, 2003.

[5] G. Deng, "Incompleteness and minimality of complex exponential system," Science in China. Series A: Mathematics, vol. 50, no. 10, pp. 1467-1476, 2007.

[6] X. Yang, "Incompleteness of exponential system in the weighted Banach space," Journal of Approximation Theory, vol. 153, no. 1, pp. 73-79, 2008.

[7] X. Yang and J. Tu, "On the completeness of the system $\left\{t^{\lambda_{n}}\right\}$ in $C_{0}(E)$," Journal of Mathematical Analysis and Applications, vol. 368, no. 2, pp. 429-437, 2010.

[8] X. D. Yang, "On the completeness of the system $t^{\lambda_{n}} \log ^{j} t$ in $C_{0}(E)$," Czechoslovak Mathematical Journal, vol. 62, pp. 361-379, 2012.

[9] X. D. Yang, "Exponential approximation of weighted Banach space on $\mathbb{R}^{n}$," Glasgow Mathematical Journal, vol. 55, pp. 115121, 2013.
[10] P. Koosis, The Logarithmic Integral, Volume I, Cambridge University Press, Cambridge, UK, 1988.

[11] P. Malliavin, "Sur quelques procédés d'extrapolation," Acta Mathematica, vol. 93, no. 1, pp. 179-255, 1955.

[12] G. Deng, "On weighted polynomial approximation with gaps," Nagoya Mathematical Journal, vol. 178, pp. 55-61, 2005.

[13] G. T. Deng, "Weighted exponential polynomial approximation," Science in China, vol. 46, pp. 280-287, 2003.

[14] N. V. Govorov, Riemann's Boundary Problem with Infinite Index, Birkhäuser, Berlin, Germany, 1994.

[15] W. Rudin, Function Theory in the Unit Ball of $\mathbb{C}^{n}$, Springer, New York, NY, USA, 1980.

[16] M. de Jeu, "Determinate multidimensional measures, the extended carleman theorem and quasi-analytic weights," The Annals of Probability, vol. 31, no. 3, pp. 1205-1227, 2003.

[17] M. de Jeu, "Subspaces with equal closure," Constructive Approximation, vol. 20, no. 1, pp. 93-157, 2004.

[18] W. Rudin, Real and Complex Analysis, China Machine Press, Beijing, China, 2003.

[19] P. Duren, Theory of $H^{p}$ Spaces, Academic Press, New York, NY, USA, 1970. 


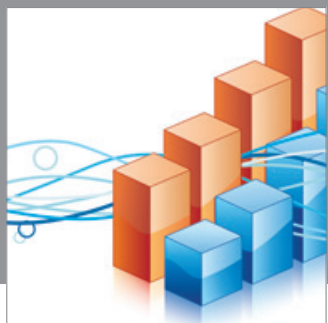

Advances in

Operations Research

mansans

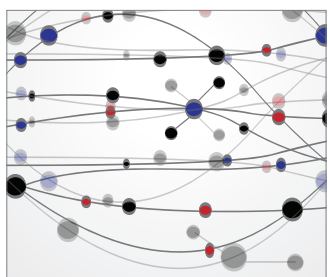

The Scientific World Journal
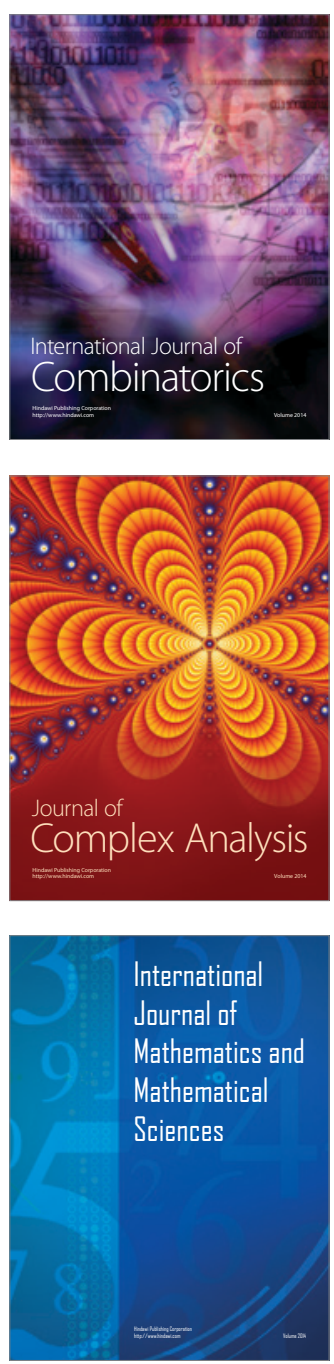
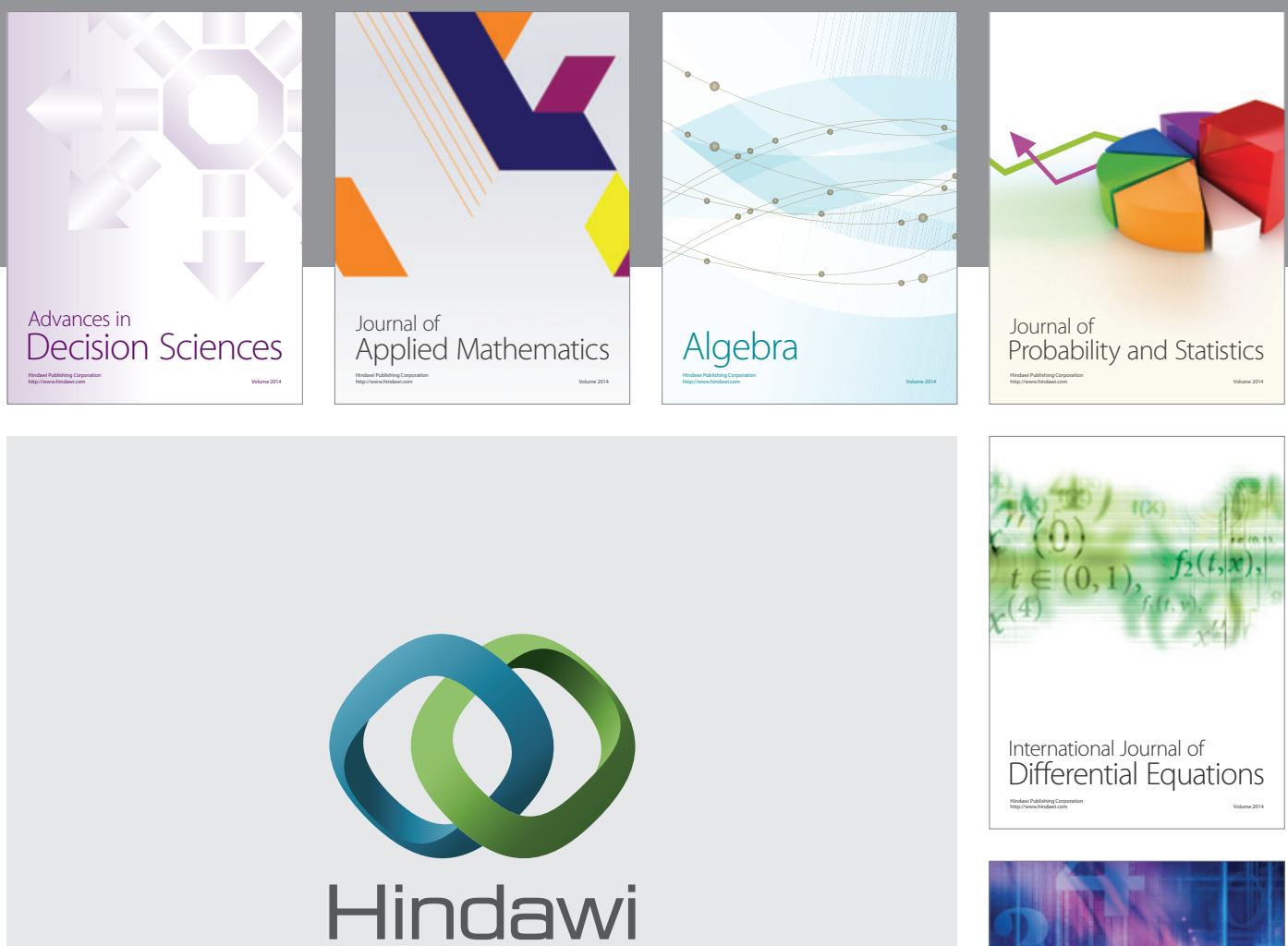

Submit your manuscripts at http://www.hindawi.com
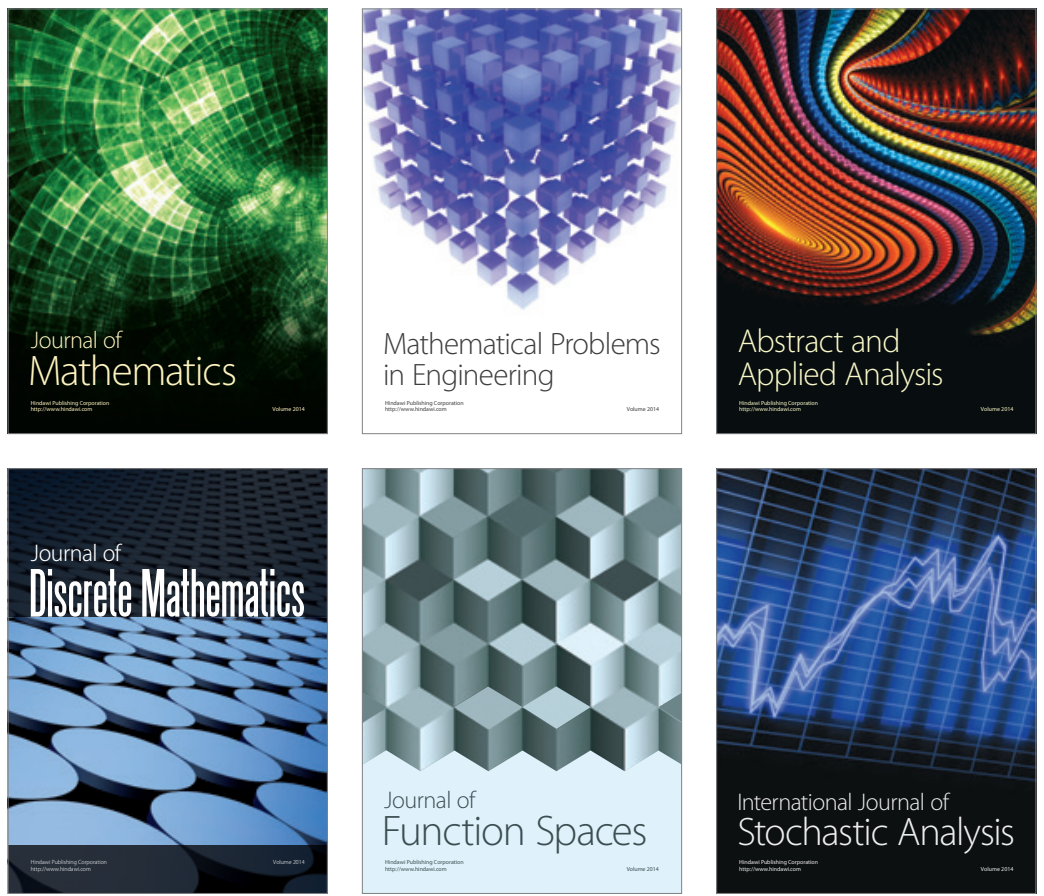

Journal of

Function Spaces

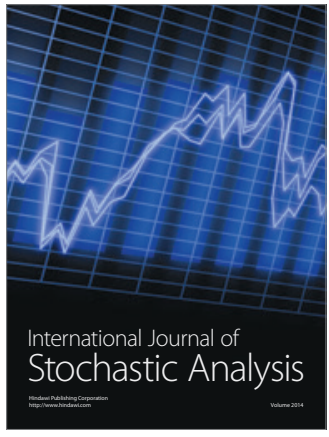

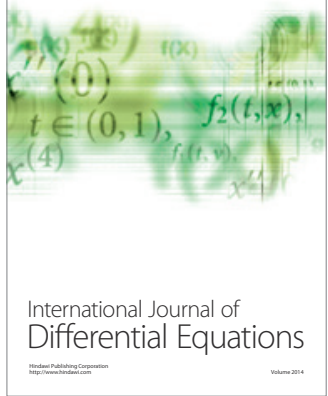
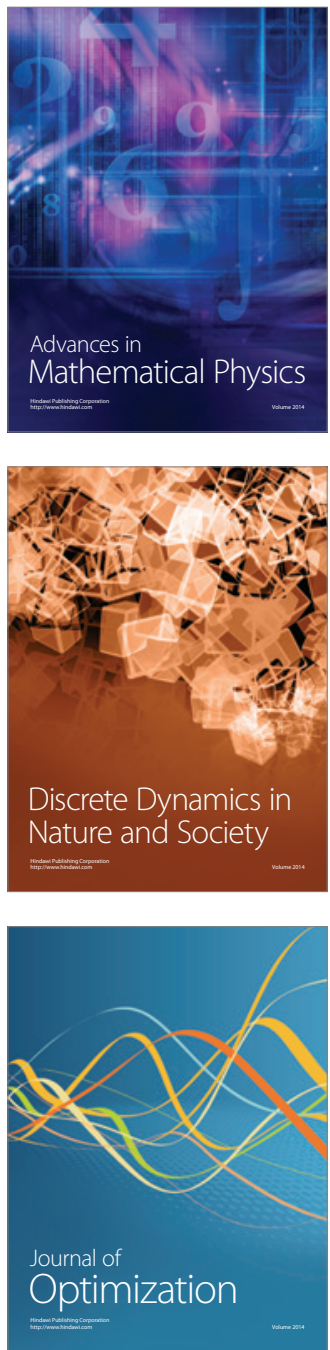